\title{
Formation of Interfacial Nanodroplets through Changes in Solvent Quality
}

\author{
Xue Hua Zhang and William Ducker* \\ Department of Chemical and Biomolecular Engineering and Particulate Fluids Processing \\ Centre, University of Melbourne, Victoria 3010, Australia.
}

Supporting Information 
Figure S1. AFM images of the interface between OTS-silicon and various aqueous solutions.

(A) Decane-saturated 40\% ethanol. The solution (3 hours old) was used to displace air from around the OTS silicon wafer. No interfacial nanodroplets were formed.

(B) Decane saturated $60 \%$ ethanol solution. The solution ( 10 hours old) was used to air from around the OTS silicon wafer. No interfacial nanodroplets were formed.

(C) Decane-saturated 40\% ethanol. The solution (6 hours old) was used to displace decane-saturated $25 \%$ ethanol (6 hours old). Some interfacial oil droplets were formed, even though we switched to a better solvent.

(D) Water. $25 \%$ ethanol ( 0.5 hour old) was displaced by water. Few and small droplets were formed.

(E) Water. Water was used to displace decane saturated 60\% ethanol solution ( $\sim 10$ hours old). Large oil droplets were formed by the exchange. 

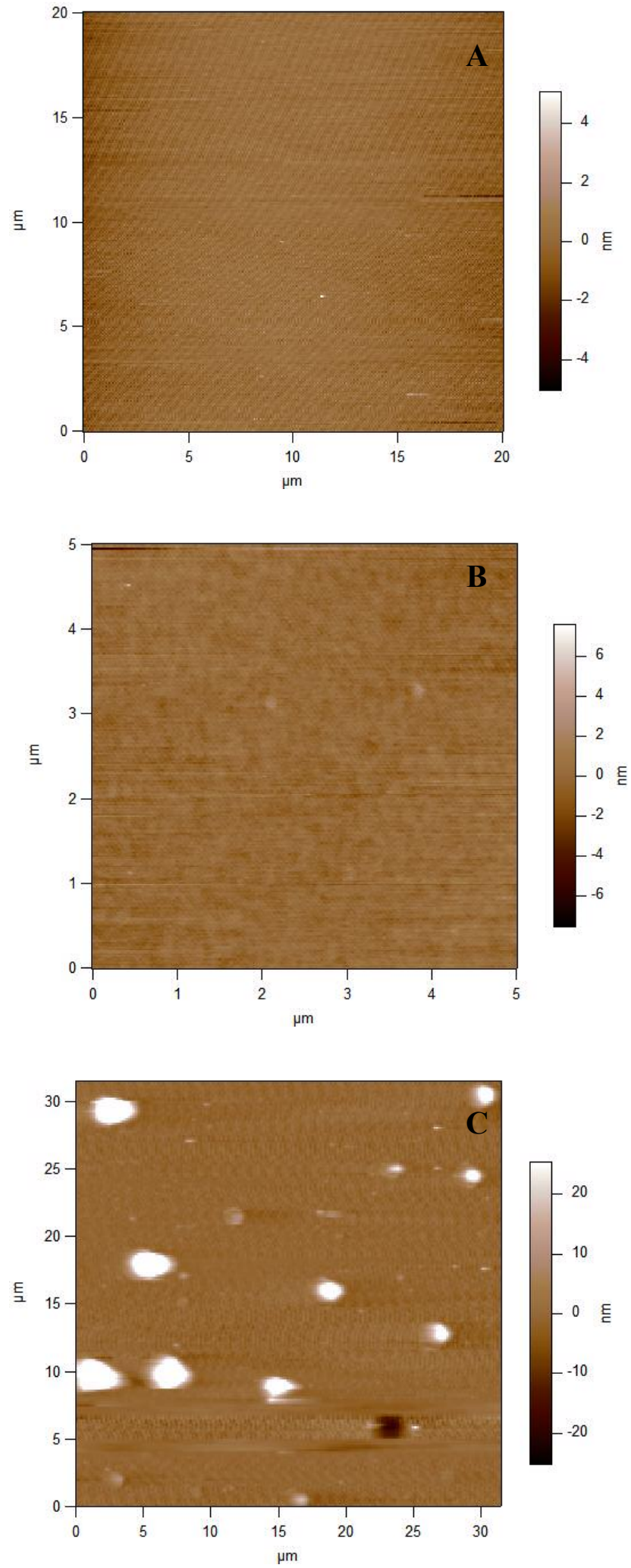

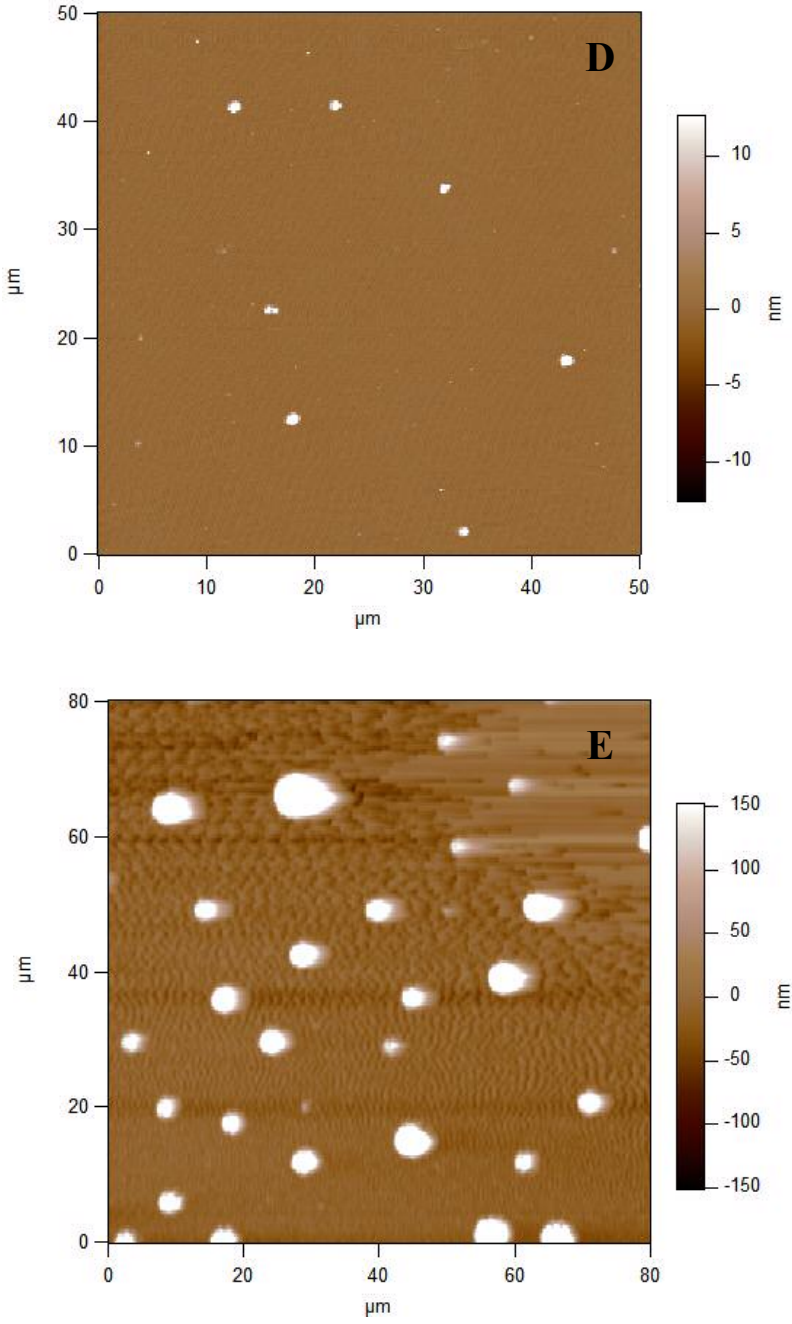
Figure S2. AFM images of the interface between highly ordered pyrolytic graphite (HOPG) and aqueous solutions

(A) decane-saturated water. The solution was used to displace decane saturated $60 \%$ ethanol (12 hours old). Many droplets with heights from tens to hundreds of nanometers were produced after the exchange.

(B) Water (without decane). Water was used to displace $60 \%$ ethanol solution (without decane). A few nanobubbles were formed by the exchange because $60 \%$ ethanol is a better solvent for air than water is for air. 

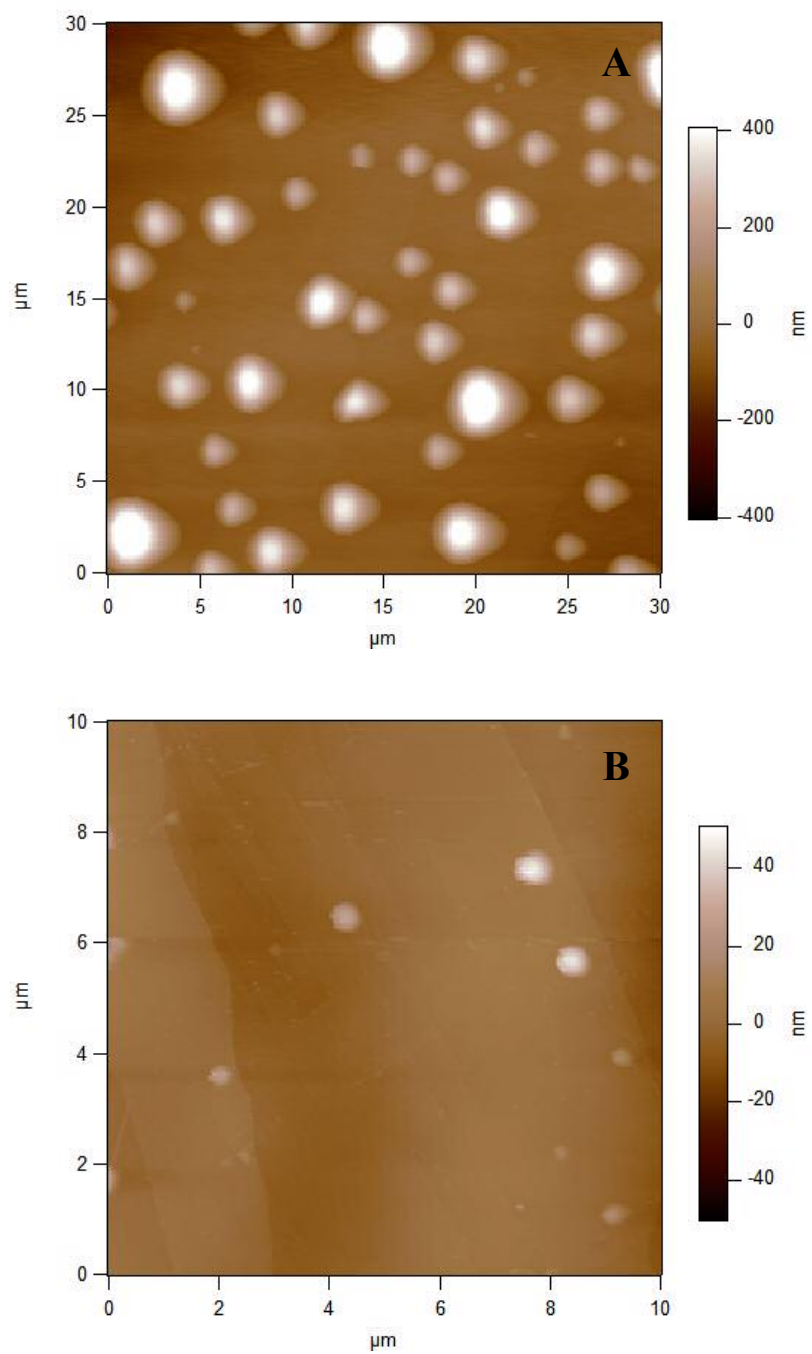
Figure S3: Effect of decane saturation on the formation of droplets on freshly cleaved HOPG.

(A) $60 \%$ ethanol solution containing decane at $20 \%$ of saturation was exchanged for water. (Image in water.) No droplets form (B) $60 \%$ ethanol solution containing decane at $80 \%$ of saturation was exchanged for water. (Image in water.) Oil droplets were produced by the exchange, even though the solution was not saturated with decane. 

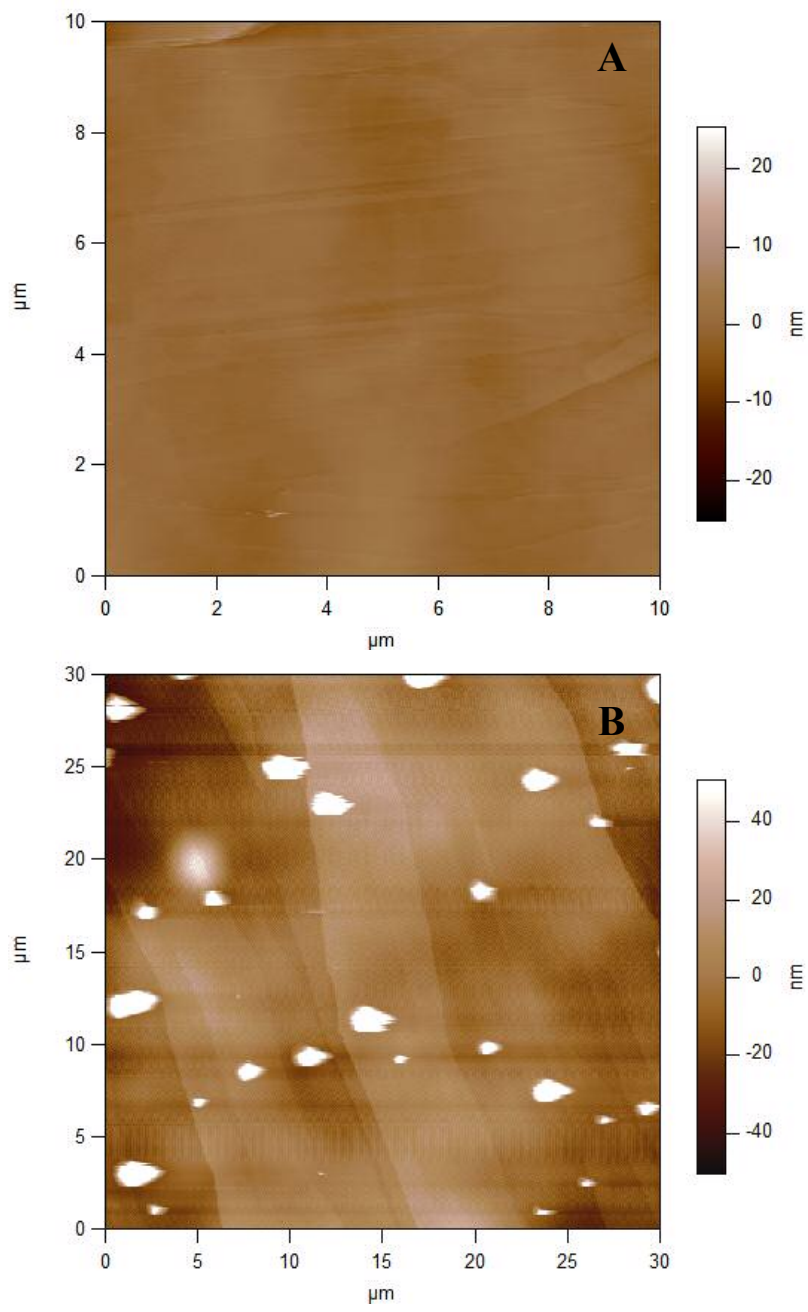